\title{
Epiphytic diatoms associated with red mangrove (Rhizophora mangle) prop roots in Bahía Magdalena, Baja California Sur, Mexico
}

\author{
D.A. Siqueiros Beltrones ${ }^{1} \&$ F.O. López Fuerte ${ }^{1}$ \\ 1 Centro Interdisciplinario de Ciencias Marinas. A.P. 592. La Paz B.C.S.; dsiquei@ipn.mx
}

Received 30-I-2001. Corrected 26-VI-2002. Accepted 11-II-2003.

\begin{abstract}
The first floristic inventory of benthic diatoms is provided for the Bahía Magdalena-Bahía Almejas lagoon system. Samplings were carried out during November of 1999. The oxydized samples were mounted permanently. Eighty six diatom taxa were identified, out of which 59 are new records for the Bahía Magdalena area, and 12 taxa are new for the Baja California peninsula. Taxa recorded previously as rare in other substrata are common or abundant on the epiphytic macroalgae of mangrove prop roots. Other species are mainly epipelic forms, while 24 are commonly found as tychoplankton in the area. Certain taxa appear to be characteristic of mangrove systems in general. Rev. Biol. Trop. 54(2): 287-297. Epub 2006 Jun 01.
\end{abstract}

Key words: benthic diatoms, epiphytes, mangrove, Bahía Magdalena, macroalgae.

Much of the epiphytic flora growing on marine macrophytes are diatoms (Kita and Harada 1962, Jacobs and Noten 1980). These may contribute significantly to the primary production of mangrove systems where diatoms from different substrata are the main microalgae forms (Siqueiros Beltrones 2002). Epiphytes enter the second level of the food chain when they are grazed by invertebrates (Navarro 1987). However, information regarding other host-epiphyte relations in mangroves is lacking, only a few floristic inventories exist, with some remarks on possible interactions (Navarro 1988), while fewer yet intent to define the structure of the epibiosis (Inclán-Rivadeneyra 1989).

Biogeographycally the Bahía Magdalena system is located within a transitional zone where tropical and temperate elements coexist (Castro Aguirre and Torres Orozco 1993). Mangroves are characteristic of the Baja California Sur coasts and B. Magdalena represents the northern limit of their distribution in the north-eastern Pacific (Blasco 1984). Mangroves constitute one of the most conspicuous tropical components of the system, and along with the ichthyological component it may have persisted since the peninsula separated from the Mexican continent six to seven m.y. ago (Castro Aguirre and Torres Orozco 1993).

Currently this area is of great importance for the fishing industry of the state. Although ichthyological studies for Bahía Magdalena are available, which support some of the most important fisheries, research on the benthic microflora that relate to other resources is lacking. Recently the microflora related to abalone fishing sites just outside the lagoon system has been undertaken (Siqueiros Beltrones 2000), but for the lagoon itself only indirect observations of tychoplanktonic diatoms have been made (Gárate Lizárraga and Siqueiros Beltrones 1998) albeit lacking taxonomic precision.

In this study we show the first results of the taxonomic survey of diatoms found on red mangrove (Rhizophora mangle L.) prop roots in order to determine which diatom species may determine the structure of the epiphytic assemblage on mangrove prop roots. 


\section{MATERIALS AND METHODS}

The sampling was carried out in Bahía Magdalena during November 1999. Twelve $30 \mathrm{~cm}^{2}$ segments were peeled off from submerged and exposed prop roots of red mangrove plants. The epiphytic macroalgae found on submerged portions of the roots were separated under a dissecting microscope. The root segments were brushed using a toothbrush rinsing with water to obtain a concentrate of sediments, organic matter and diatoms that were observed in fresh mounts to determine the proportion of live cells. The diatom frustules from the roots and macroalgae were cleaned by oxydizing the organic matter using nitric acid and alcohol. Permanent preparations were mounted by triplicate using Meltmount (R.I. = 1.7) (Siqueiros Beltrones 2000).

Drawings were made of the observed taxa on curatorial cards which include information on location, habitat, frequency, and meristic and morphometric data. These were included along with the slides in the diatom collection (Diatomario) of the Museo de Historia Natural of the Universidad Autónoma de Baja California Sur. Microphotographs of representative taxa were taken under an Olympus CH-2 microscope with phase contrast illumination.

Taxonomic determinations were done following the classic works of Peragallo and Peragallo (1897-1908), Hustedt (1955, 1959, 1961-1966), Hendey (1964), Cleve-Euler (1968), McIntire and Reimer (1974), Foged (1975, 1984), Navarro (1982), Siqueiros Beltrones and Ibarra Obando (1985), Simonsen (1987), Moreno et al. (1996), Siqueiros-Beltrones and Sánchez Castrejón (1999), Siqueiros-Beltrones and Morzaria Luna (1999), Siqueiros Beltrones (2000), Siqueiros Beltrones (2002). Round et al. (1990) was used for updating generic nomenclature. The epiphytic macroalgae were identified following Abbot and Hollenberg (1976).

Study Area. Bahía Magdalena is a shallow lagoon located on the western coast of Baja California Sur, between $24^{\circ} 15^{\prime}-25^{\circ} 20^{\prime} \mathrm{N}$ and $111^{\circ} 30^{\prime}-112^{\circ} 12^{\prime} \mathrm{W}$ (Fig. 1). It is part of the
Bahía Magdalena-Bahía Almejas lagoon system (Gárate Lizárraga and Siqueiros Beltrones 1998). The middle part of the Bahía Magdalena littoral where the sampling point was located (San Carlos docking area) is densely populated by mangroves mainly Rhizophora mangle and Avicennia germinans (black mangrove) bordering the coastline.

\section{RESULTS}

A total of 86 diatom taxa were identified as epiphytes of Rhizophora mangle prop roots (Table 1). 59 taxa are new records for the system.

Most of the taxa in this study were better represented on the macroalgae (Ulva sp., 1 species of the gelidiales, and a Polysiphonia species) separated from the root segments which appeared heavily epiphytized. In vivo observations of macroalgal thalli showed the presence of multispecific colonial growths in mucilaginous films (Amphora bigibba, Amphora proteus, Amphora ventricosa, Caloneis elongata, Cocconeis dirupta, Cocconeis scutellum,

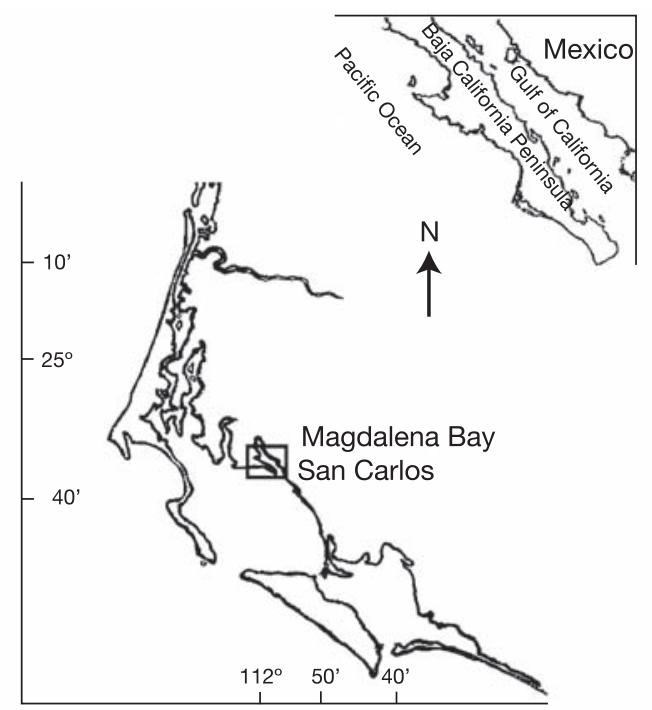

Fig. 1. Location of the Bahía Magdalena mangrove system and sampling site. 
TABLE 1

Epiphytic diatoms collected on red mangrove (Rhizophora mangle) prop roots in Bahia Magdalena,

B.C.S., during November 1999

Achnanthes brevipes var. Intermedia (Kütz.) Cleve E, T, O

Rare

Achnanthes delicatula (Kütz.) Grunow E

Rare

Achnanthes hauckiana Grunow

Abundant

Achnanthes kuwaitensis Hendey E

Common

Achnanthes manifera Brun E

Rare

Achnanthes pseudogroenlandica var. Phineyii E

Common

Achnanthes yaquinensis McIntire \& Reimer E

Common

Actinoptychus aster Brun T

Rare

Actinoptychus vulgaris Schuman T, O

Rare

Amphora angusta Gregory E

Rare

Amphora bigibba Grunow E, T, O

* Amphora clevei Grunow

Amphora coffeaeformis var. salina (Wm. Smith) A. Cleve

Abundant

Rare

* Amphora obtusa var. typica Paragallo \& Paragallo T, O

Common

* Amphora ocellata Donkin E, T

Rare

Amphora proteus Cleve E, T

Rare

Amphora strigosa Hustedt E

Common

Amphora ventricosa (Greg.) Cleve T, O

Common

Amphora sp. 1

* Auliscus punctatus Bailey

* Caloneis elongata (Grun.) Boyer E

Caloneis linearis (Grun.) Boyer

Campylodiscus thuretii Brébisson

Catenula pelagica Mereshkowsky T, O

Cocconeis dirupta Gregory $\mathrm{O}$

Cocconeis placentula Ehrenberg

* Cocconeis pseudomarginata Gregory

Cocconeis scutellum Ehrenberg E

Cocconeis scutellum var. Parva Grun. Ex. Cleve E

Cyclotella litoralis Lange \& Syvertsen E, T, O

Denticula kuetzingii Grunow

Rare

Rare

Rare

Common

Common

Common

Abundant

Rare

Rare

Abundant

Abundant

Abundant

Rare

Denticula subtilis Grunow

Dimeregramma maculatum (Cleve) Frenguelli

Dimeregramma minor var. Genuina A. Cleve

Diploneis gravelleana Hagelstein T, O

Diploneis gruendleri (A. Schmidt) Cleve

Diploneis obliqua (Brun.) Hustedt T, O

Diploneis suborbicularis var. Intermedia A. Cleve

Fallacia nummularia (Grev.) Mann T, O

Common

Abundant

Rare

Common

Rare

Common

Rare

Rare

Common

Fallacia approximatoides (Hust.) Mann

Common

Fallacia vittata (Cleve) Mann

Gomphonemopsis pseudoexigua (Simonsen) Medlin E

Common

Rare

Grammatophora marina Ehrenberg

Lyrella clavata var. subconstricta (Greg.) Mann T, O

Common

Lyrella exsul (A.S.) Mann

Melosira nummuloides (Dillwyn) Agardh E, T

Common

Abundant

Rare

Rare

Navicula cancellata Donkin

* Navicula contenta Grunow E

* Navicula ergadensis Gregory T

Abundant

Rare

* Navicula gastrum (Ehr.) Donkin

Rare

Navicula pennata Schmmidt E, T, O

Abundant

Navicula platyventris Meister E

Navicula salinarum Grunow E

Common

Nitzschia angularis Wm. Smith

Common

Rare 
TABLE 1 (Continued)

Epiphytic diatoms collected on red mangrove (Rhizophora mangle) prop roots in Bahía Magdalena,

B.C.S., during November 1999

Nitzschia brittoni Hagelstein

Nitzschia dissipata (Kütz.) Grunow

Nitzschia frustulum var. perminuta Grunow

Nitzschia grossestriata Hustedt

Nitzschia lanceolata var. minor Grunow

Nitzschia obtusa var. brevissima Grunow

Nitzschia sigma Wm. Smith

Nitzschia socialis Gregory

Nitzschia subhybrida Hustedt

Nitzschia subtilis Grunow T, O

Odontinium marinum Grunow $\mathrm{O}$

Opephora pacifica (Grun.) Petit

Paralia sulacata (Ehr.) Cleve E, T, O

Paralia sulacata var. crenulata Grunow T, O

Paralia sulcata var. radiata (Grun.) Paragallo \& Paragallo T, O

Petroneis granulata (Bail) Mann

Plagiotropis vitrea var. genuina A. Cleve

Psammodictyon constrictum (Kütz.) D.G. Mann

Psammodictyon panduriformis var. lata (Greg.) Mann

Seminavis sp.

Staurophora amphioxys (Greg.) Mann

Staurosirella pinnata (Ehr.) Mann

Surirella fastuosa Ehrenberg

Surirella fastuosa var. cuneata A. Schmidt

Synedra tabulata var. affinis (Kütz.) A. Cleve

* Synedra gailloni Ehrenberg E

Terpsinoe americana (Bail.) Ralfs

* Thallassiosira leptopus (Grunow) Hasle \& Fryxell E, O

Trachyneis aspera Ehrenberg E, T, O

Trachyneis clepsydra (Donk.) Cleve E

* Trachyneis velata (A.S.) Cleve T, O

Tryblionella acuminata W. Smith T, O
Rare

Abundant

Rare

Common

Common

Common

Rare

Rare

Common

Common

Common

Abundant

Common

Common

Rare

Rare

Rare

Abundant

Rare

Common

Rare

Common

Common

Common

Abundant

Abundant

Rare

Rare

Rare

Common

Common

Common

Notes: * New records for the area.

$\mathrm{E}=$ Previously recorded as epiphytic on mangrove prop roots or as abundant in this study; $\mathrm{T}=$ recorded also as tychoplankton; $\mathrm{O}=$ also collected offshore.

Cocconeis scutellum var. parva and Trachyneis spp.), stalked chains (Achnanthes yaquinensis, Cyclotella stylorum, Melosira nummuloides, Paralia sulcata), or erect tufts of Synedra gaillonii (Fig. 2a, b, c, respectively).

Taxa such as Navicula contenta, Navicula platyventris, Navicula pennata and Navicula salinarum and most of the Achnanthes species had been recorded elsewhere as epiphytes (Table 1) either from macroalgae or mangrove prop roots (Navarro 1987). Caloneis elongata, Navicula contenta, Navicula platyventris, Psammodyction constricta, Synedra gaillonii, and Trachyneis velata were abundant in our samples, although they are recorded for the first time for the peninsula, along with 6 other taxa (Table 1).

A representative array of the diatom taxocoenosis in the study site is presented in Figs. 3-6. 

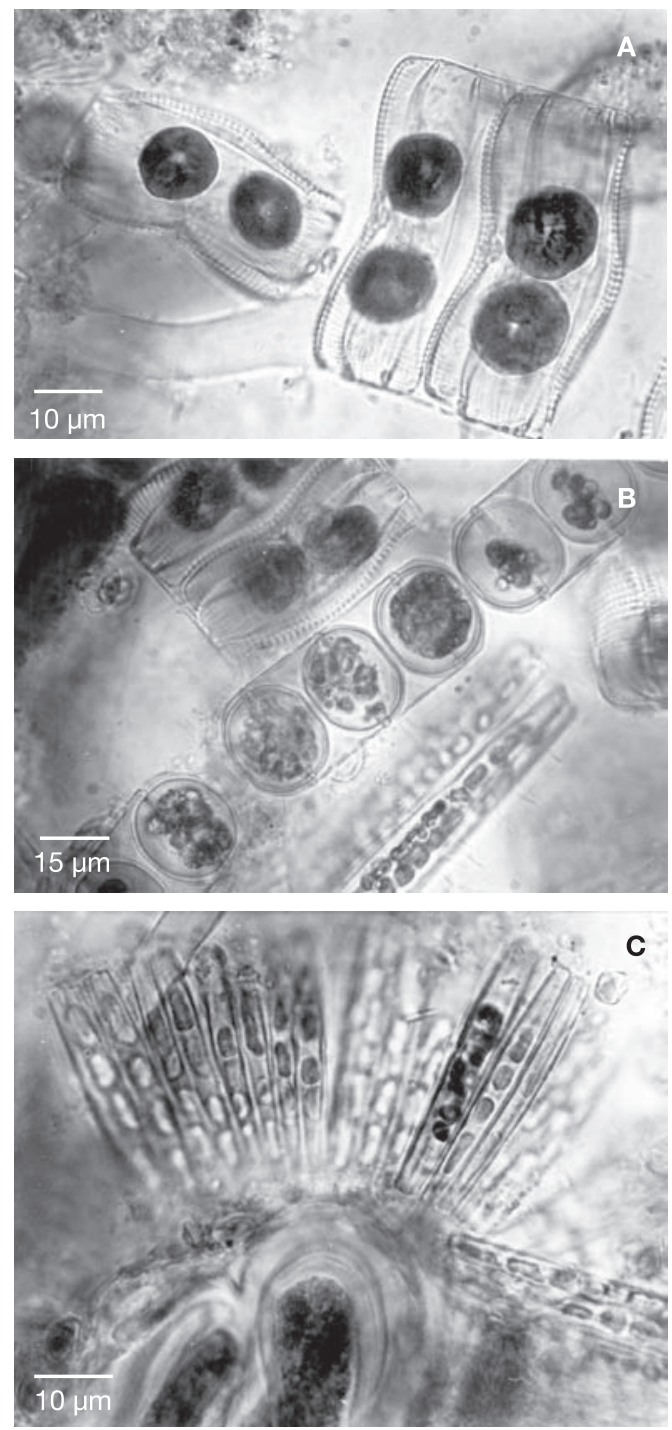

Fig. 2. Most conspicuous diatoms growing on epiphytic macroalgae collected from mangrove prop roots: a) Stalked chains of Achnanthes yaquinensis; b) Chains of Melosira nummuloides; c) Erect colonies of Synedra gailloni.

\section{DISCUSSION}

Previous studies on benthic diatoms in the system comprising only the rocky environment just outside Bahía Magdalena account for the remaining 27 taxa recorded in the system (Siqueiros Beltrones 2000, Siqueiros Beltrones and Valenzuela Romero 2001).
On the other hand, otherwise rare taxa from previous records in the region such as Achnanthes yaquinensis, Amphora bigibba, Cyclotella stylorum, and Melosira nummuloides (Siqueiros Beltrones 2002), were either common or abundant. Such observations have been made also on Macrocystis pyrifera in undergoing studies (Siqueiros Beltrones et al. 2002). All this indicates that host-epiphyte relations are important for the growth of certain benthic diatoms and some may be specific, mainly of the epiphytic macroalgae, but apparently not for the mangrove roots. However, the specificity of the macroalgae-prop root relation should be analyzed in order to determine if an indirect association exists between diatoms and mangrove roots.

Several species, although not abundant occur frequently enough to be considered epiphytes (e.g. Amphora proteus, Amphora ocellata, Caloneis elongata, Caloneis linearis) but further observations are required to confirm this. Other taxa, such as Diploneis obliqua, Lyrella exsul, Petroneis granulata, Surirella fastuosa and Surirella fastuosa var. cuneata occur principally in sediments of mangrove systems (SiqueirosBeltrones and Sánchez-Castrejón 1999). These species may be opportunistic colonizers from the bottom sediments that migrate upward on the sediments covering the prop roots.

The epiphytic or epipelic taxa from the B.C.S. mangrove systems are somewhat similar to those from mangroves in different parts of the world (Foged 1975, 1984, Navarro 1987) and Sinaloa, México (Moreno et al. 1996, Siqueiros Beltrones and Martínez López unpublished.). The current inventory of benthic diatoms from mangrove systems, including this paper, has yielded 181 new records altogether for the Baja California peninsula since 1999 (Siqueiros-Beltrones and Sánchez Castrejón 1999, Siqueiros-Beltrones and Morzaria Luna 1999). Although the northern limit for most of them is not known certain taxa appear to be characteristic of the mangrove environment and have not been observed in other localities along the peninsula, especially on the northern part (Siqueiros Beltrones 2002). 

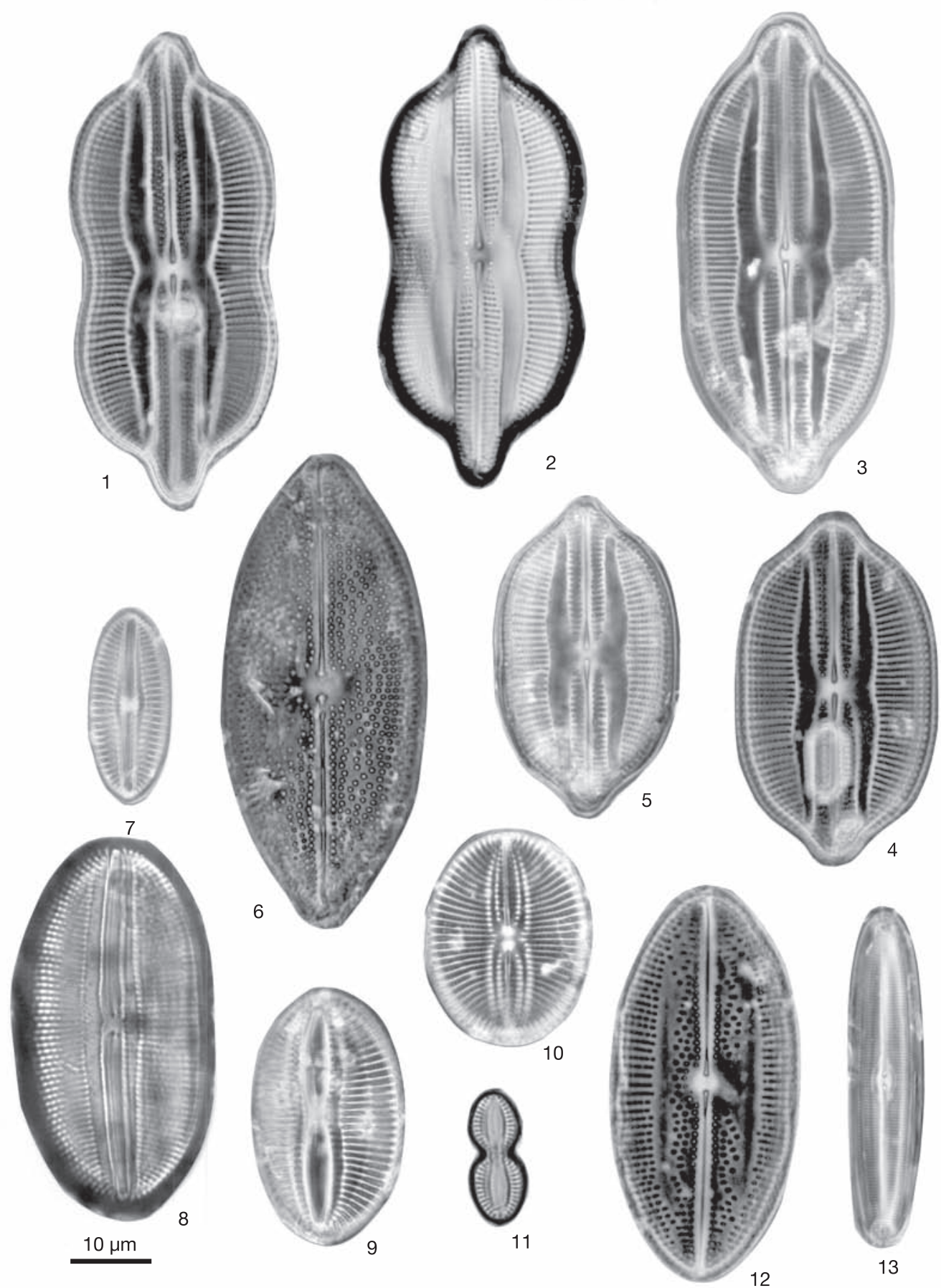

Fig. 3. 1) Lyrella exsul (A. Schmidt) Mann, 2) Lyrella exsul (A. Schmidt) Mann, 3) Lyrella clavata (Greg.) D.G. Mann, 4) Lyrella clavata var. indica (Grun.) Cleve, 5) Lyrella clavata (Greg.) D.G. Mann, 6) Petroneis granulata (Bailey) Mann, 7) Diploneis subovalis Cleve, 8) Diploneis obliqua (Brun) Hustedt, 9) Diploneis suborbicularis var. intermedia A. Cleve, 10) Fallacia nummularia (Greg.) Mann, 11) Diploneis gravelleana Hagelstein, 12) Fallacia approximatoides (Hust.) Mann, 13) Caloneis elongata (Grun.) Boyer. 


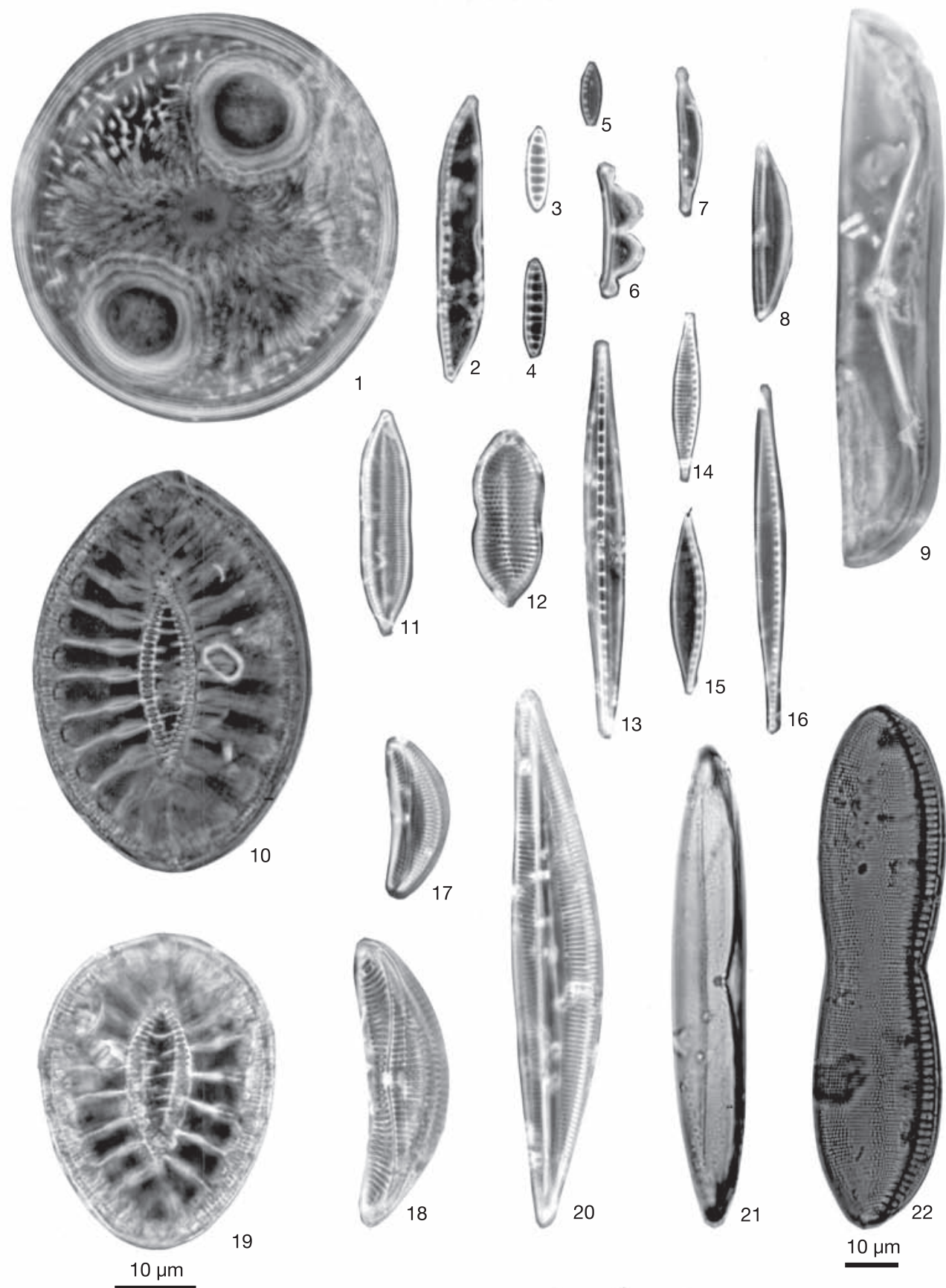

Fig. 4. 1) Auliscus punctatus Bailey, 2) Nitzschia obtusa var. brevissima Grunow, 3) Odontinium marinum Grunow, 4) Denticula subtilis Grunow, 5) Nitzschia frustulum var. perminuta Grunow, 6) Amphora bigibba Grunow, 7) Amphora sp. 1, 8) Amphora strigosa Hustedt, 9) Amphora obtusa var. typica Peragallo \& Peragallo, 10) Surirella fastuosa Ehrenberg, 11) Tryblionella acuminata W. Smith, 12) Psammodyction constrictum (Kütz.) Mann, 13) Nitzschia angularis Wm. Smith, 14) Nitzschia grossestriata Hustedt, 15) Nitzschia lanceolata var. minor Grunow, 16) Nitzschia subtilis Grunow, 17-18) Amphora proteus Cleve, 19) Surirella fastuosa var. cuneata A. Schmidt, 20) Amphora ventricosa (Greg.) Cleve, 21) Plagiotropis vitrea var. genuina A. Cleve, 22) Psammodyction panduriformis var. lata (Greg.) Mann. 

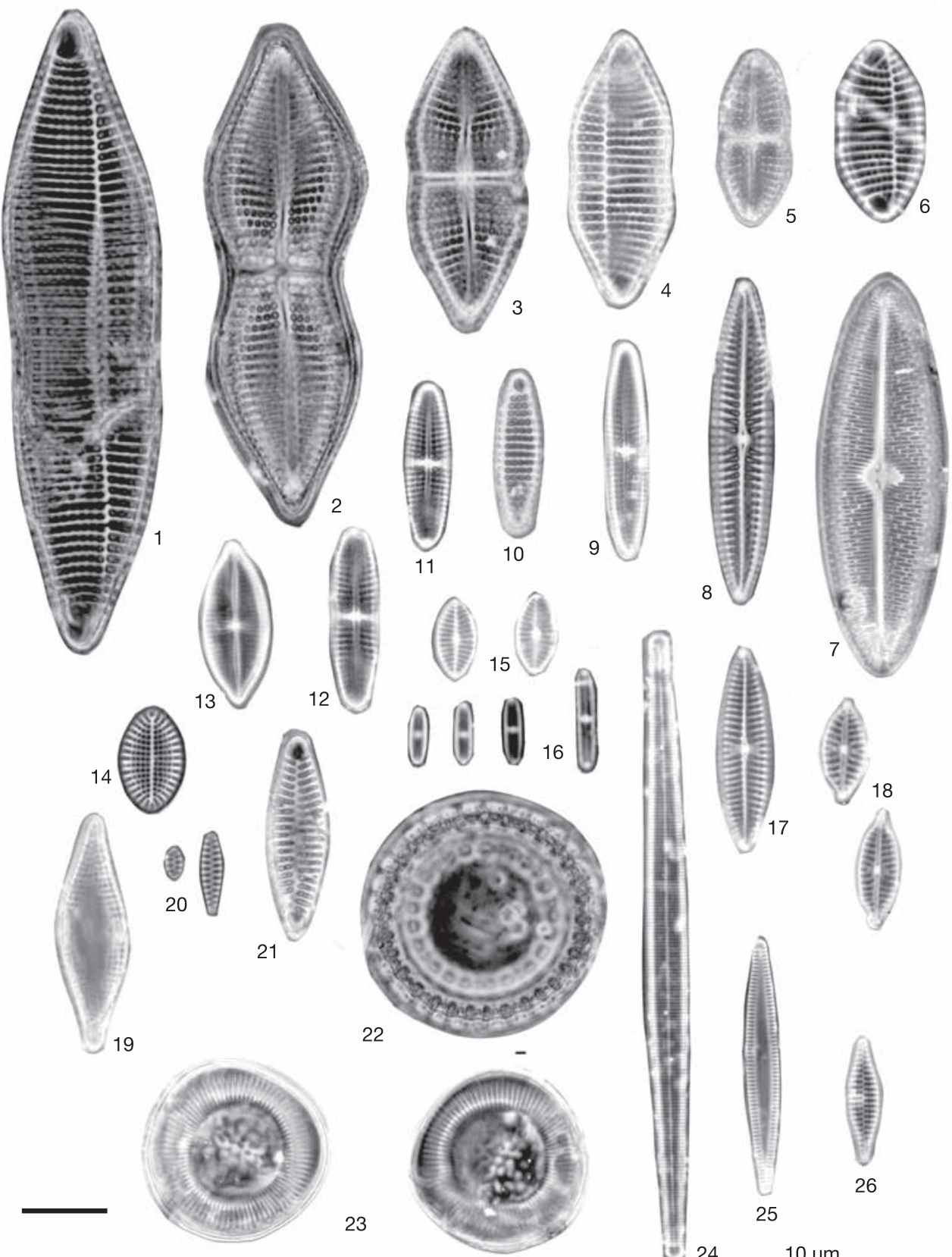

22

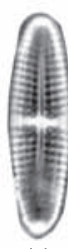

11

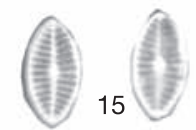

(1) - 16 递

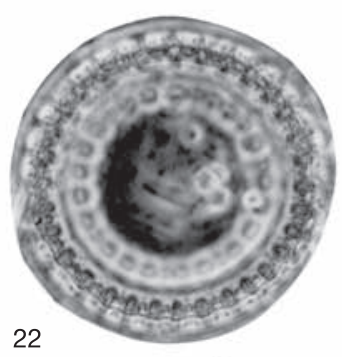

-

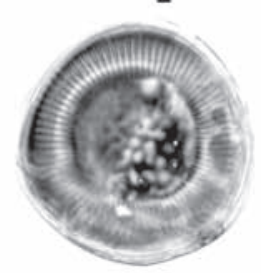

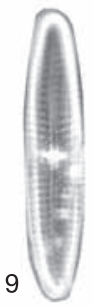

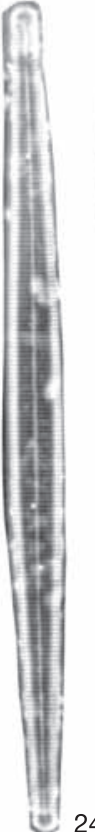

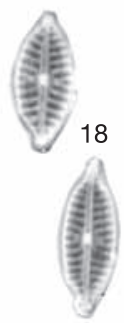
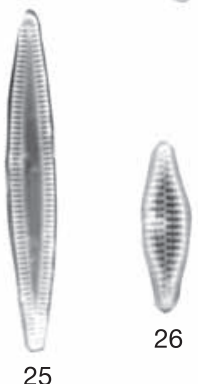

25

24

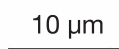

Fig. 5. 1-6) Achnanthes yaquinensis McIntire \& Reimer, 7) Trachyneis velata (A.Schmidt) Cleve, 8) Navicula pennata Schmidt, 9) Achnanthes pseudogroenlandica var. phinneyii McIntire \& Reimer, 10) Achnanthes kuwaitensis Hendey, 11) Achnanthes kuwaitensis Hendey, 12) Achnanthes brevipes var. intermedia (Kütz.) Cleve, 13) Achnanthes manifera Brun, 14) Cocconeis scutellum var. parva Grunow ex Cleve, 15) Achnanthes hauckiana Grunow, 16) Navicula contenta Grunow, 17) Navicula pennata Schmidt, 18) Navicula platyventris Meister, 19) Dimeregramma maculatum (Cleve) Frenguelli, 20) Opephora pacifica (Grun.) Petit, 21) Dimeregrama minor var. genuina A. Cleve, 22) Paralia sulcata (Ehr.) Cleve, 23) Cyclotella litoralis Lange \& Syvertsen 1,24) Synedra gailloni Ehrenberg, 25) Synedra tabulata var. affinis (Kütz.) A. Cleve, 26) Neodelphineis pelagica Takano. 

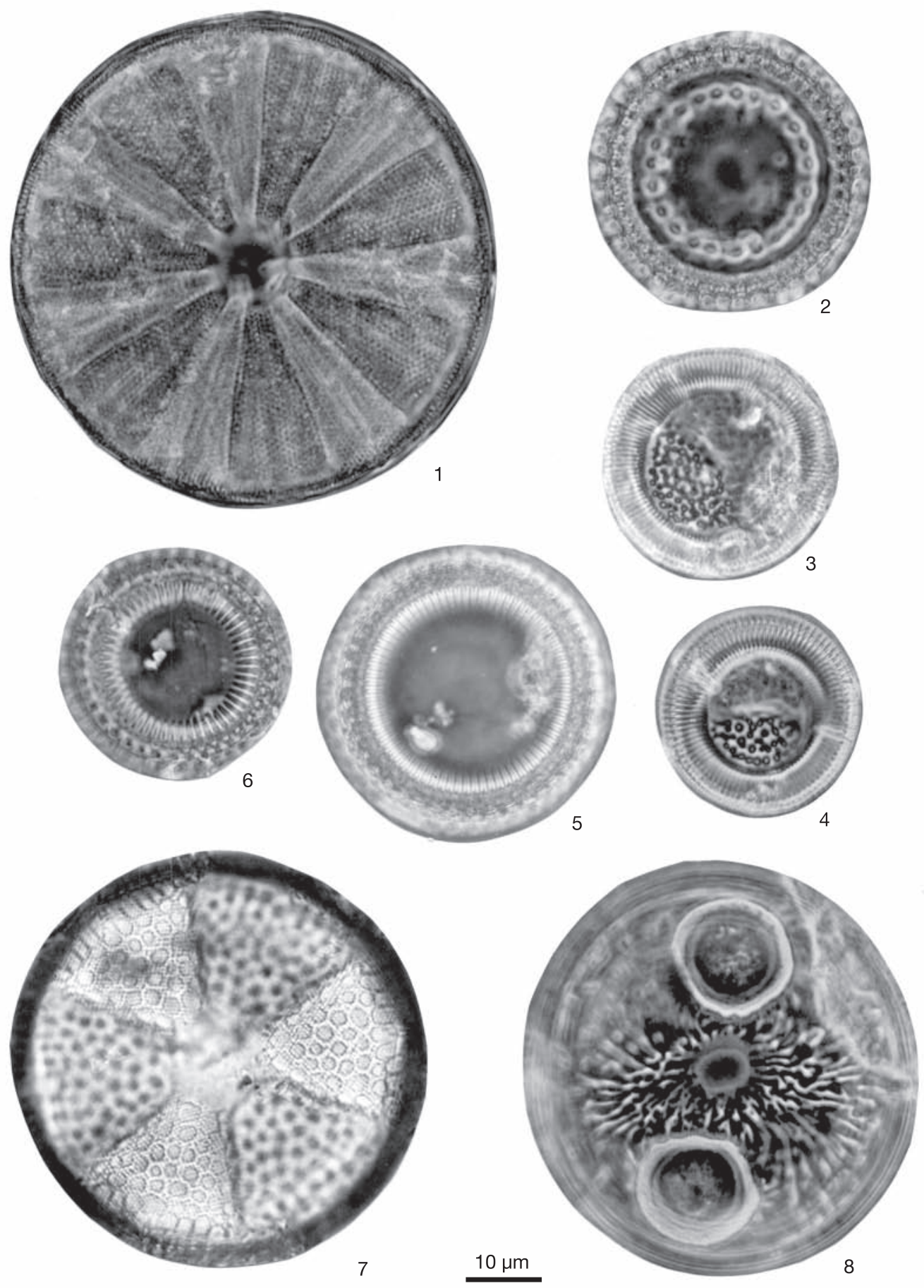

Fig. 6. 1) Actinoptychus vulgaris Schuman, 2) Paralia sulcata (Ehr.) Cleve, 3) Cyclotella litoralis Lange \& Syvertsen, 4) Cyclotella litoralis Lange \& Syvertsen, 5) Paralia sulcata var. radiata (Grunow) Peragallo \& Peragallo, 6) Paralia sulcata var. radiata (Grunow) Peragallo \& Peragallo, 7) Actinoptychus aster Brun, 8) Auliscus punctatus Bailey. 
Twenty four taxa from our inventory (Table 1) have been collected previously in phytoplankton tows (tychoplankton) representing an important part of the estimated biomass (Gárate Lizárraga and Siqueiros Beltrones 1998). Although the species were not then identified, we observed them recently in samples collected by the above authors at the northern mouth of the Bahía Magdalena system, and can be considered as common tychoplankton. Also, 21 of the benthic diatom taxa in this study (Table 1) have been collected in deep water traps deployed $300 \mathrm{~m}$ offshore north-west of Bahía Magdalena. The occurrence of benthic diatoms in the traps and in the samples from phytoplankton tows suggests that organic material is exported offshore through the northern opening of the system (Martínez López et al. 2004).

Tidal mixing has been identified as an important factor causing the presence of abundant benthic diatoms in the water column (Gárate Lizárraga and Siqueiros Beltrones 1998). However, much work has to be done to complete the floristic inventory and analyze the association structure of benthic diatoms from the Bahía Magdalena-Bahía Almejas lagoon system. This information may provide important tools to explain oceanographic, ecological, and biogeographical phenomena in the area.

\section{ACKNOWLEDGMENTS}

The sampling for this study was carried out during field trips for the Microalgas Marinas course of the Departamento de Biología Marina of the Universidad Autónoma de Baja California Sur. The Museo de Historia Natural of the Universidad Autónoma de Baja California Sur financed laboratory material for processing the samples. Elisa Serviere from the Centro de Investigaciones Biológicas del Noroeste aided in the identification of the macroalgae.

\section{RESUMEN}

Se presenta el primer inventario florístico de diatomeas bénticas para el Sistema Lagunar Bahía Magdalena-Bahía
Almejas. Se hicieron muestreos en noviembre de 1999. En las muestras oxidadas y montadas permanentemente, se identificaron 86 táxones, de los cuales 59 son nuevos registros para el área de Bahía Magdalena, y 12 son nuevos para la península de Baja California. Táxones previamente registrados como escasos en otros sustratos son comunes o abundantes sobre macroalgas epífitas de raíces primarias de mangle. Otras species son principalmente formas epipélicas, mientras que 24 son constituyentes comunes del ticoplancton del área. Ciertos táxones parecen ser característicos de sistemas de manglar en general.

Palabras clave: diatomeas bentónicas, epífitas, mangle, Bahía Magdalena, macroalgas.

\section{REFERENCES}

Abbot, I.A. \& G.J. Hollenberg. 1976. Marine algae of California. Standford University, Stanford, California, USA. 827 p.

Blasco, F. 1984. Climatic factors and the biology of mangrove plants. pp. 18-35. In Snedaker, S. \& J. Snedaker (eds.). The mangrove ecosystem. Research Methods. UNESCO/SCOR. 525 p.

Castro Aguirre, J.L. \& R. Torres Orozco. 1993. Consideraciones acerca de Bahía MagdalenaAlmejas, un sistema lagunar de la costa occidental de Baja California Sur, México. An. Esc. Nac. Cienc. Biol., México 38: 67-73.

Cleve-Euler, A. 1968. Die diatomeen von schweeden un Finnland. In V. Kramer (ed.). Bibliotheca Phycologica, Band 5. Wesley, New York, USA. 963 p.

Foged, N. 1975. Some littoral diatoms from the coast of Tanzania. Bibliotheca Phycologica, Band 16. Kramer, Vaduz, Germany. 123 p., 31 plates.

Foged, N. 1984. Freshwater and littoral diatoms from Cuba. Bibliotheca Diatomologica, Kramer, Germany. 123 p., 60 plates.

Gárate Lizárraga, I. \& D.A. Siqueiros Beltrones. 1998. Time variations in phytoplankton assemblages in a subtropical lagoon system after the 1982/83 El Niño event (1984/86). Pac. Sci. 52(1): 79-97.

Hendey, N.I. 1964. An introductory account of the smaller algae of Brittish coastal waters. Part V: Bacillarophyceae (Diatoms). Fisheries Investigation Series IV: HMSO, London. 317 p.

Hustedt, F. 1955. Marine littoral diatoms of Beaufort North Carolina. Duke University Mar. Station Bull. 6: 1-67. 
Hustedt, F. 1959. Die kieselalgen Deutschland, Osterreichs un der Schweiz. In L. Rabenhorst (ed.). KryptogamenFlora von Deutschland, Osterreich un Schweiz, VII Band, II Teil. Koeltz Scientific Books (Rep. 1991), Germany. 845 p.

Hustedt, F. 1961-66. Die kieselalgen Deutschland, Osterreichs un der Schweiz. In L. Rabenhorst (ed.). Kryptogamen-Flora von Deutschland, Osterreich un Schweiz, VII Band, III Teil. Koeltz Scientific Books (Rep. 1991), Germany. 816 p.

Inclán-Rivadeneyra, R. 1989. Ecología de la epibiosis en las raíces inmersas de Rhizophora mangle en Bahía de la Asención Quintana Roo, México. Cienc. Mar. 15(1): 1-20.

Jacobs, R.P.W.M. \& T.M.P.A. Noten. 1980. The annual pattern of the diatoms in the epiphyton of eelgrass (Zostera marina) at Roscoff, France. Aquat. Bot. 8: $355-370$

Kita, T. \& E. Harada. 1962. Studies on the epiphytic communities. 1. Abundance and distribution of microalgae and small animals on the Zostera marina blades. Pub. Seto Mar. Biology Lab. 10(2): 245-257.

Martínez López, A. \& D.A. Siqueiros Beltrones \& N. Silverberg. Martínez López, A. \& D.A. Siqueiros Beltrones \& N. Silverberg. 2004. Transport of benthic diatoms across the continental shelf of southern Baja California Peninsula. Cien. Mar. 30(4): 503-513.

McIntire, C.D. \& C.W. Reimer. 1974. Some Marine and Brackish-Water Achnanthes from Yaquina Estuary, Oregon (U.S.A.). Bot. Mar. 17: 164-175.

Moreno, J.L., S. Licea \& H. Santoyo. 1996. Diatomeas del Golfo de California. Universidad Autónoma de Baja California Sur-SEP-Promarco. México. 273 p.

Navarro, N.J. 1982. Marine diatoms associated with mangrove prop roots in the Indian River, Florida, USA. J. Cramer. Germany. $151 \mathrm{p}$

Navarro, N.J. 1987. Diatomeas epífitas de Puerto Rico I. Grammatophora oceanica Ehr. var. oceanica. Science-Ciencia 14(1): 13-18.
Navarro, N.J. 1988. Diatomeas en tapetes microbianos de Puerto Rico. Gayana Botanica 45(1-4): 275-281.

Round, F.E., R.M. Crawford \& D.G. Mann. 1990. The diatoms. Cambridge University, Cambridge, U.K. $747 \mathrm{p}$.

Peragallo, H. \& M. Peragallo. 1897-1908. Diatomées marines de France et districts maritimes voisins. M.J. Tempere, France. 491 p.

Simonsen, R. 1987. Atlas and catalogue of the diatom types of F. Hustedt I, II, II. J. Cramer. Berlin, Germany.

Siqueiros-Beltrones, D.A. 2000. Benthic diatoms associated to abalone (Haliotis spp.) on a rocky substratum from Isla Magdalena, Baja California Sur, México. Oceánides 15(1): 35-46.

Siqueiros Beltrones, D.A. 2002. Diatomeas bentónicas de la península de Baja California; diversidad y potencial ecológico. Oceánides, Centro Interdisciplinario de Ciencias Marinas / IPN - Universidad Autónoma de Baja California Sur, México. 102 p., 43 láminas.

Siqueiros-Beltrones, D.A. \& S.E. Ibarra-Obando. 1985. Lista florística de las diatomeas epífitas de Zostera marina en Bahía Falsa, San Quintín, B.C. Cienc. Mar. 11(3): 21-67.

Siqueiros Beltrones, D.A. \& H. Morzaria Luna. 1999. New records of marine benthic diatoms for the north-western Mexican region. Oceánides 14(2): 89-95.

Siqueiros Beltrones, D.A. \& E. Sánchez Castrejón. 1999. Association structure of benthic diatoms from a mangrove environment in a Mexican subtropical lagoon. Biotropica 31: 48-70.

Siqueiros Beltrones, D.A. \& G. Valenzuela Romero. 2001. New records of benthic diatoms from natural grazing surfaces of abalone (Haliotis spp.) habitats in the Baja California peninsula. Oceanides 16(2): 107-126.

Siqueiros Beltrones, D.A., E. Serviere-Zaragoza \& U. Argumedo Hernández. 2002. Epiphytic diatoms of Macrocystis pyrifera (L.) Agardh from the Baja California peninsula. Oceánides 17(1): 31-39. 
